\title{
REVISIÓN
}

\section{Cáncer de colon y su relación con la fortificación de ácido fólico}

\author{
Marion Guerrero-Wyss ${ }^{a, *}$, Yudixia Vásquez-Pincheira ${ }^{a}$ \\ a \\ Facultad de Ciencias de la Salud, Universidad San Sebastián, Chile. \\ *marion.guerrero@uss.cl
}

Recibido el 31 de octubre de 2015; aceptado el 12 de mayo de 2016.

Cáncer de colon y su relación con la fortificación de ácido fólico

\section{PALABRAS CLAVE RESUMEN}

Cáncer de colón;

Cáncer colorrectal;

Ácido fólico;

Alimentos fortificados.

La fortificación de la harina de panificación en Chile con ácido fólico en mujeres embarazadas redujo en forma significativa los defectos del tubo neural, sin embargo esta situación también afecta al resto de la población. ¿Qué sucede con la suplementación en niños, adultos y adultos mayores que no requieren un aporte extra de ácido fólico? El objetivo de esta revisión es relacionar la fortificación de ácido fólico con el riesgo de desarrollar cáncer de colon. Algunos metaanálisis indican que la suplementación con folatos a largo plazo no aumentaría el riesgo relativo e incidencia de cáncer colorrectal, no obstante otros estudios creen pertinente estimar factible esta relación, ya que los datos no son concluyentes. La bibliografía disponible presenta resultados contradictorios; dependiendo del diseño metodológico del estudio, fuente de folato, dosis de fortificación y duración de ésta, además de la población objetivo; por lo que estas características deben ser consideradas en el análisis del riesgo de desarrollo de cáncer colorrectal. Se debe considerar que las pruebas no son concluyentes en relación a suplementación de folatos y el riesgo de desarrollar cáncer colorrectal. Los datos indican que la relación es dependiente de dosis y tiempo de administración, diseño metodológico, lugar geográfico del estudio, etnia de los sujetos, y otras variables como estilos de vida pudieron influir en algunos estudios. Se recomienda analizar futuros estudios realizados a nivel local y seguir con cautela la incidencia de cáncer colorrectal y relacionarlo con el desarrollo del programa de fortificación nacional con ácido fólico. 
Colon cancer and the relationship with folic acid fortification

\section{KEYWORDS}

Colonic Neoplasms;

Colorectal

Neoplasms;

Folic Acid;

Food, Fortified.

\section{ABSTRACT}

The fortification of flour baking in Chile with folic acid in pregnant women significantly reduced the neural tube defects, however this situation also affects the rest of the population. What about supplementation in children, adults and adults older that do not require an extra intake of folic acid? Relate folic acid fortification with the risk of developing colon cancer is the aim of this review. Some meta-analyzes indicate that folate supplementation does not increase long-term relative risk and incidence of colorectal cancer, however other relevant studies indicate this relationship to estimate feasible because the evidence is inconclusive. The available evidence shows conflicting results; depending on the methodological study design, source of folate, fortification dose and duration of this addition to the target population; so these features should be considered in the analysis of the risk of developing colorectal cancer. It should be considered that the evidence is inconclusive regarding folate supplementation and the risk of developing colorectal cancer. Evidence indicates that the relationship is dependent on dose and time of administration, methodological design, geographical location of the study, subject ethnicity, and other variables such as lifestyle may have influenced some studies. It is recommended to analyze future studies locally and follow carefully the incidence of colorectal cancer and relate national program development folic acid fortification.

\section{CITA}

Guerrero-Wyss M, Vásquez-Pincheira Y. Cáncer de colon y su relación con la fortificación de ácido fólico. Rev Esp Nutr Hum Diet. 2016; 20(3): 236 - 243. doi: 10.14306/renhyd.20.3.191

\section{INTRODUCCIÓN}

El programa de fortificación de harina de trigo ${ }^{1,2}$ se estableció como obligatorio desde enero del año 2000 (2,0-2,4mg de ácido fólico $/ \mathrm{kg}$ ), siendo su principal objetivo aumentar la ingesta de folatos, especialmente en mujeres en edad fértil ( $400 \mathrm{mg} /$ día) y reducir la prevalencia de defectos del tubo neural.

La harina de trigo fue seleccionada como vehículo de fortificación ${ }^{3}$, debido a que el pan y sus derivados, corresponden a alimentos de consumo habitual en Chile y su adición no altera las características organolépticas de estos productos $^{2}$. Esta intervención ha sido altamente costo-efectiva ${ }^{4,5}$, determinando una importante reducción en la prevalencia de nacimientos con defectos del tubo neural durante los últimos años.

Sin embargo, la existencia de una mayor oferta de alimentos fortificados ha generado preocupación a nivel internacional ${ }^{6}$ y un creciente interés por evaluar la existencia de riesgos asociados a su exceso en grupos que no se benefician directamente con esta intervención.

Estudios recientes señalan que la suplementación con ácido fólico efectuada antes del desarrollo de una neoplasia suprimiría su desarrollo y progresión ${ }^{6}$, no obstante favorecería su desarrollo cuando ésta ya existe. Cáncer de colon, así como cáncer de mama y próstata, podrían relacionarse con ingestas elevadas de folatos, especialmente, como ácido fólico.

La mayor ingesta de ácido fólico se puede desencadenar por niveles de fortificación superiores a la recomendación ${ }^{6}$ y por mayor consumo de alimentos fortificados o suplementos nutricionales que determinarían una mayor probabilidad de exceder la ingesta máxima tolerable o upper level (UL).

El principal objetivo de este estudio es relacionar la fortificación de ácido fólico con el riesgo de desarrollar cáncer de colon, en población objeto de esta fortificación. 


\section{RELACIÓN ENTRE ÁCIDO FÓLICO Y CÁNCER COLORRECTAL}

\section{Fortificación de alimentos y límites máximos de ácido fólico}

Un estudio realizado el año $2010^{6}$, analizó la fortificación de la harina de trigo con ácido fólico en Chile con el objetivo de conocer la existencia de poblaciones en riesgo por ingestas excesivas de dicho nutriente. La información sobre niveles de ácido fólico en harina se solicitó a la Autoridad Sanitaria: Departamento de Nutrición y Alimentos, Secretarías Regionales Ministeriales (SEREMIs) y al Instituto de Salud Pública (ISP) a través del portal Chile Transparente. Los antecedentes de niveles de folatos sanguíneos se recopilaron de artículos chilenos y de información obtenida de algunos talleres efectuados en el MINSAL. El consumo de pan promedio estimado en niños fue extraído de una publicación nacional.

Se calculó la ingesta de ácido fólico proveniente del consumo aparente de pan, considerando un contenido de ácido fólico en harinas según valores de los percentiles (p) 20, 50 y 95 de cada distribución anual. Para cálculos, se consideró un pan promedio de $100 \mathrm{~g}$ conteniendo $87 \mathrm{~g}$ de harina. El aporte de ácido fólico proveniente de otros alimentos no fue incluido en el análisis.

Se observó que entre los años 2005 y 2007, el consumo de 2 a 3 unidades de pan/día elaborados con harina con niveles de ácido fólico sobre el p95 permitiría alcanzar ingestas superiores al UL.

En escolares de nivel socioeconómico bajo, se observó que quienes presentaban consumo diario de pan sobre p75 podrían tener ingestas cercanas al UL $(600 \mathrm{mg} / \mathrm{d})^{6}$. Durante la pre-fortificación, sólo el $42,7 \%$ de las mujeres presentaba ingestas superiores al EAR (requerimiento promedio estimado, por sus siglas en inglés), aumentando a $99 \%$ en el período post-fortificación, con ingestas promedios 3,5 veces superior al EAR, mientras que el 2,3\% de ellas presentaba ingestas cercanas al UL6

Los datos del estudio anterior ${ }^{6}$, que consideraba adultos mayores, permitieron estimar que el $82 \%$ presentaba ingesta de EDF (equivalente dietario de folato) superiores al EAR. En este mismo grupo se observó un aumento durante la etapa post-fortificación, detectándose que el 100\% presentaba ingesta superior al EAR. Considerando indicadores de folato sérico en un estudio desarrollado con posterioridad ${ }^{7}$, se observó que la ingesta de EDF estimada en adultos varones fue 3,2 veces superior al EAR, mientras que el $6 \%$ de ellos presentaba ingestas superiores al UL.
Los resultados de este estudio indicaron la necesidad de realizar una evaluación de riesgos integrada de este programa de fortificación, considerando costos, beneficios y eventuales riesgos o peligros, con el fin de adecuar la dosis de fortificación en harinas considerando que existe un porcentaje importante de mujeres, niños y adultos con ingestas de ácido fólico cercanas al UL. El principio de beneficencia implicaría adoptar medidas necesarias para optimizar beneficios, minimizando riesgos, y reducir la sobrexposición innecesaria en algunos grupos, pero asegurando los beneficios en embarazadas.

\section{Ingesta de ácido fólico y riesgo de cáncer de colon}

Disminución del riesgo de cáncer de colon: Durante el año 2002 se realizó un análisis de caso-cohorte de 56.837 mujeres $^{8}$ que se inscribieron en el Canadian National Breast Screening Study y que completaron un cuestionario dietético auto administrado. Durante el seguimiento a finales del año 1993, un total de 389 mujeres fueron diagnosticadas con cáncer colorrectal, identificado por la base de datos Canadian Cancer. Para fines comparativos, una subcohorte de 5.681 mujeres fue seleccionada al azar. Después de exclusiones, por diversas razones, el análisis se basó en 295 casos y 5.334 controles.

La ingesta de folato se asoció inversamente al riesgo de cáncer colorrectal. Entre los individuos con baja ingesta de metionina y folato no pareció disminuir el riesgo de cáncer rectal; esto podría explicarse, en parte, al bajo número de casos en el análisis de subgrupos. En general, los resultados se inclinaron para apoyar la hipótesis que una elevada ingesta de folato se asocia a menor riesgo de cáncer colorrectal.

Los resultados sugirieron que la ingesta de folato en la dieta disminuye el riesgo de cáncer de colon, aunque la tendencia no fue muy clara 8 .

En el año 2010, se publicó un metaánálisis de ensayos de intervención ${ }^{9}$ para determinar suplementación con ácido fólico y su relación en la prevención de adenomas colorrectales, debido a los datos contradictorios de estudios clínicos previos. El análisis mostró que la suplementación con ácido fólico no tuvo efectos protectores sobre la recurrencia de los adenomas colorrectales (odds ratio =1,08 [IC del 95\%; 0,87; $1,33 ; p=0,49])$, ni tiene un resultado positivo en el número de pólipos recurrentes por paciente $(p=0,41)$. El efecto general para todos los estudios incluidos no fue significativo (odds ratio $=0,78$ [IC del 95\%; 0,49; 1,24; $p=0,30]$ ). El presente metaanálisis no ha demostrado beneficio potencial para la administración de suplementos de ácido fólico ${ }^{9}$. 
Otro estudio realizado durante el $2012^{10}$, describiendo en una revisión sistemática vía PubMed de estudios clínicos randomizados, controlados, doble ciego y sus referencias, que evaluaron específicamente el efecto de suplementación de ácido fólico sobre la recurrencia de adenomas colorrectales; en la revisión se incluyeron siete estudios y de acuerdo al análisis no fue posible concluir que la suplementación de ácido fólico presente efectos beneficiosos sobre la recurrencia de adenoma colorrectal. Cabe destacar que en algunos estudios hubo diferencias de riesgo según el tipo de folato administrado, muestra que sugiere revisar los criterios y niveles de suplementación en algunos subgrupos de población con mayor riesgo ${ }^{10}$.

Un metaanálisis publicado el año $2013^{11}$ estudió la administración de suplementos de ácido fólico y el riesgo relativo de cáncer a través de ensayos clínicos aleatorios. En general, no se observó efecto significativo sobre la incidencia total de cáncer ( 13 ensayos, $n=49.406$; $R R=1,05$; IC del 95\%: 0,99 a 1,11; $p=0,13)$, así como tampoco se modificó el riesgo relativo de cáncer de próstata, pulmón, colorrectal, mama, neoplasia hematológica y la mortalidad total por cáncer. Sin embargo, se observó una reducción significativa del riesgo de melanoma (tres ensayos, $n=19.128 ; 0,47 ; 0,23$ a 0,94 ; $p=0,03)^{11}$.

Otro estudio realizado el año $2013^{12}$ consideró un análisis combinado de tres grandes ensayos aleatorios de suplementos de ácido fólico para la prevención de adenomas metacrónicos en 2.632 pacientes con un historial de adenoma. Hubo una tendencia estadística no significativa de la disminución del riesgo de adenoma asociado con suplementos de ácido fólico con el aumento de ingesta de alcohol.

Durante los primeros años de seguimiento informados, más muertes ocurrieron en el grupo placebo que en el grupo de ácido fólico ( $1,7 \%$ vs. 0,5\%; $p=0,002)$. En conclusión, después hasta 3,5 años de uso de ácido fólico, no hay una disminución clara o aumento en la aparición de nuevos adenomas en pacientes con una historia de adenoma ${ }^{12}$.

\section{Aumento del riesgo de cáncer de colon}

En una cohorte prospectiva realizada el año $2001^{13}$, participaron 88.756 enfermeras registradas en Estados Unidos (30 a 55 años), Nurses' Health Study. Las cuales completaron un cuestionario semicuantitativo de frecuencia de consumo que incluía información sobre 61 alimentos y bebidas, además de suplementos de vitaminas y minerales. Se calcularon los riesgos relativos y ajustados por ingesta de energía, edad, historia familiar de cáncer colorrectal, consumo de aspirina, tabaquismo, masa corporal, e ingesta de carne roja, fibra y metionina.
El seguimiento de la cohorte fue casi total (96\%) y se identificaron 442 nuevos casos de adenocarcinoma de colon. La ingesta superior de folato total ajustado estaba relacionado con un menor riesgo de cáncer de colon para la ingesta de $>400 \mathrm{mg} /$ día en comparación con $<200 \mathrm{mg} /$ día. La ingesta de folato dietético se relacionó con una reducción moderada del riesgo, mientras que sólo mujeres que usaban suplementos durante 15 años o más presentaron una reducción importante del riesgo ${ }^{13}$.

Un estudio realizado durante el año $2004^{14}$, fue un metaanálisis de publicación de datos para proporcionar una estimación global de la asociación de la dieta y la ingesta total de folato (folato de alimentos y suplementos) con el riesgo de cáncer colorrectal (CCR). Se consideraron artículos publicados en PubMed desde el año 1950 hasta Enero de 2004, de acuerdo al análisis se concluyó que la asociación entre ingesta de ácido fólico y riesgo de CCR no difieren significativamente, según la fuente de folato (la dieta o total) o de acuerdo al sexo.

Los resultados de este metaanálisis orientan a la hipótesis que el consumo relativamente elevado de folato se asocia con una disminución en el riesgo de CCR, pero se confunden por otros factores dietéticos los cuales no es posible descar$\operatorname{tar}$ (consumo de vitaminas, fibra, etc.) ${ }^{14}$. Se necesitan estudios adicionales para determinar si el folato contenido en alimentos o el folato contenido en suplementos se asocian a mayor reducción del riesgo.

En un estudio realizado en Finlandia ${ }^{15}$, se relacionó el nivel de ácido fólico y el cáncer colorrectal centrado en el efecto del $\alpha$-tocoferol y el $\beta$-caroteno de varones fumadores de 50-69 años. El ácido fólico se evaluó en 144 casos (91 con cáncer de colon, 53 cáncer de recto) y 276 controles. La base de la dieta de ácido fólico estaba disponible en un cuestionario alimenticio para 386 de estos sujetos (96\%). Los participantes con alto consumo de alcohol, bajos índices de ácido fólico y una dieta baja en proteínas se asociaban a mayor riesgo de desarrollar cáncer de colon que quienes mantenían un bajo consumo de alcohol, altos índices de ácido fólico y una dieta alta en proteínas. Finalmente, no se observó una asociación significativa entre el ácido fólico y cáncer de colon en varones fumadores en dicho estudio ${ }^{15}$.

En 2007 se realizó un estudio basado en la hipótesis entre asociación de fortificación de ácido fólico y el aumento de prevalencia de cáncer de colon $^{16}$. La fortificación a nivel nacional de los granos de cereales con ácido fólico se inició en Estados Unidos y Canadá en 1996 y 1997, respectivamente, y fue de carácter obligatorio en 1998. El fundamento fue reducir el número de nacimientos complicados por defectos del tubo neural. Al mismo tiempo, ambos países 
experimentaron retrocesos bruscos de la tendencia a la disminución de cáncer colorrectal ${ }^{16}$. La baja incidencia de cáncer colorrectal en décadas anteriores, comenzó a aumentar en 1996 (Estados Unidos) y 1998 (Canadá), alcanzando su punto máximo en 1998 (Estados Unidos) y 2000 (Canadá). Estas observaciones no prueban causalidad, pero son consistentes con los efectos conocidos de folato en neoplasias existentes, como se encuentra descrito en estudios preclínicos y clínicos. Por lo tanto, la hipótesis de que la fortificación con ácido fólico puede haber sido total o parcialmente responsable del aumento de las tasas de cáncer colorrectal a finales de los años 90, eventualmente pudiera ser real.

La explicación del aumento de las tasas de cáncer colorrectal entre 1996 y 2000 y su relación con la fortificación de ácido fólico es materia de especulación basada en una asociación temporal entre estos dos fenómenos. La literatura al respecto indica que la alta ingesta de ácido fólico puede acelerar el crecimiento de tumores previamente existentes.

Una investigación realizada el año $2008^{17}$, buscaba determinar asociación entre suplementación con ácido fólico y reducción de prevalencia de adenoma colorrectal y sus precursores. Se realizó un ensayo controlado con placebo, doble ciego en pacientes con pólipos adenomatosos en Detroit (VA Medical Center), los pacientes con adenoma colorrectal fueron asignados al azar para recibir una dosis de $5 \mathrm{mg}$ al día de ácido fólico o placebo durante 3 años. De los 137 sujetos seleccionados después de la confirmación histológica de pólipos, sólo 94 completaron el estudio; 49 conformaron el grupo suplementado y 45 el grupo control. El promedio de pólipos recurrentes a los 3 años fue 0,36 para el grupo de pacientes suplementados con ácido fólico en comparación con 0,82 para los sujetos tratados con placebo, reflejando un aumento de la prevalencia de pólipos 3 veces superior en el grupo control versus grupo intervenido. Aquellos pacientes menores de 70 años de edad y con adenomas colónicos o adenomas en etapa avanzada respondieron mejor al suplemento de ácido fólico ${ }^{17}$.

Estos resultados sugieren una posible asociación entre la baja ingesta de ácido fólico y la necesidad de estudios más amplios que evalúen el aporte dietético de ácido fólico y metionina, e indicadores bioquímicos de ácido fólico (concentración de ácido fólico en eritrocitos y suero), homocisteína y vitamina $B_{12}$. Por lo tanto, se sostiene con los datos actuales y hasta que existan nuevas publicaciones para definir la naturaleza de esta relación, que esta aseveración debería ser considerada en el debate y discusión de los programas de fortificación de ácido fólico en países que presentan un incremento de la prevalencia de adenomas colorrectales.
El año 2013 se realizó una importante revisión sistemática de la literatura y metaanálisis de estudios de cohorte prospectiva sobre el uso de suplementos de la dieta y el riesgo de cáncer colorrectal18. Los metaanálisis de estudios observacionales sugirieron un papel beneficioso para las multivitaminas y suplementos de calcio sobre el riesgo de cáncer colorrectal, mientras que la asociación con otros suplementos y el riesgo de cáncer colorrectal es inconsistente, pudiendo incluso afectar a éste último variables confundentes como estilo de vida. Los autores concluyeron la necesidad de una extensa evaluación del uso de suplemento dietético y una mejor comprensión de los mecanismos subyacentes ${ }^{18}$.

Debido a los datos poco claros disponibles, un grupo de autores $^{19}$ decidió realizar un metaánalisis incluyendo ocho ensayos clínicos controlados randomizados publicados en PubMed y EMBASE antes de octubre de 2014. Los resultados indicaron que el tratamiento con ácido fólico no se asoció con el riesgo de cáncer colorrectal en la población total (riesgo relativo $[R R]=1,00 ; 95 \%$ intervalo de confianza $[I C]=0,82$ a 1,$22 ; p=0,974)$. Sin embargo, los investigadores sugieren validar este hallazgo con otros estudios a largo plazo y con mayor tamaño muestral ${ }^{19}$.

Se evaluaron efectos de la suplementación de ácido fólico en la incidencia global y específica de cáncer a través de un metaanálisis de ensayos clínicos randomizados con un $\mathrm{n}=50.000$ individuos ${ }^{20}$. Se consideró ensayos que suplementaban durante 2 a 5 años con ácido fólico en dosis superiores a las habitualmente utilizadas en la fortificación de harinas, y el resultado fue que los suplementos de ácido fólico no modificaron la incidencia de cáncer en sitios específicos ${ }^{20}$.

\section{Influencia de polimorfismos en la asociación de ingesta de folatos y riesgo de cáncer de colon}

En un metaanálisis publicado el año $2012^{21}$, se incluyeron 67 estudios observacionales en los cuales se evaluó el riesgo de los polimorfismos metilentetrahidrofolato reductasa (MTHFR), niveles de ingesta de folatos asociados y riesgo de cáncer colorrectal. Los resultados sugirieron que el genotipo 677TT se asoció con un riesgo reducido de desarrollar cáncer colorrectal, en condiciones de ingesta total elevada de áci-

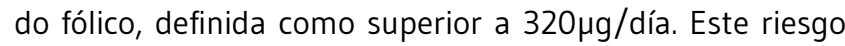
asociado permanecería reducido tanto para MTHFR 677CC como para genotipo TT. No obstante los autores concluyen que son necesarios más estudios sobre el efecto dosis dependiente de la ingesta de ácido fólico y su asociación en el impacto de riesgo de cáncer colorrectal en presencia de los polimorfismos descritos en este estudio ${ }^{21}$. 
Otro metaanálisis realizado también durante el 2012 incluyó 21 estudios de 6.692 casos y 8.266 controles $^{22}$, el objetivo fue identificar asociación entre el polimorfismo MTHFR C677T y el riesgo de cáncer colorrectal en poblaciones asiáticas, ya que los estudios anteriores individualmente presentaban resultados contradictorios. En los resultados destaca que hubo una asociación del alelo MTHFR 677T con un menor riesgo de cáncer colorrectal $(\mathrm{OR}=0,91$; IC 95\%=0,85 a 0,98; $\mathrm{p}=0,011$ ) en población asiática y no hubo relación de acuerdo al efecto de folato en plasma de la población ${ }^{22}$.

En otro metaanálisis publicado el año $2013^{23}$, se determinó asociación entre el polimorfismo C1420T en el gen de la serina hidroximetiltransferasa citosólica (SHMT1) y riesgo de cáncer colorrectal. Se evaluaron los datos individuales sobre 5.043 casos y 6.311 controles de 15 estudios de casos y controles publicados. Los autores concluyeron que en general no hubo asociación, pero sugirieron efectos de geografía específica del polimorfismo SHMT1 que hacen a europeos susceptibles, pero no a estadounidenses. El nivel de folato parece mostrar una asociación inversa de este polimorfismo con el cáncer colorrectal ${ }^{23}$.

\section{Discusión}

La bibliografía disponible sobre la ingesta de ácido fólico y su relación con el desarrollo de cáncer colorrectal es contradictoria. Se debe diferenciar para el análisis en los estudios la fuente de folatos; si corresponde a dieta o suplemento, ya que podría modificar el riesgo de adenoma de colon. También se debe considerar la estructura química de los folatos y el ácido fólico, ya que por corresponder a compuestos diferentes se metabolizan por vías distintas. Cabe señalar, que el aumento del riesgo de cáncer colorrectal puede modificarse no sólo por la ingesta, sino también el tiempo de extensión de su consumo. Del mismo modo, se puede establecer una clara diferencia en los resultados y variación del riesgo de cáncer de colon de acuerdo a área geográfica y también etnia asociada. Otra variable importante a considerar es el tipo de estudio, ya que existe bibliografía asociada a cohortes y también a estudios de casos y controles con un gran tamaño muestral.

También cabe destacar que si bien no fue el objetivo de esta revisión, en la literatura se describen asociaciones entre la ingesta de folatos con modificaciones del riesgo de otros tipos de cáncer como pancreático ${ }^{24,25}$, cáncer de próstata ${ }^{26,27}$, cáncer de cavidad oral y faringe ${ }^{28}$, cáncer mamario ${ }^{29-33}$, cáncer pulmonar ${ }^{34,35}$, cáncer de vejiga ${ }^{36}$, cáncer gastrointestinal ${ }^{37}$, por lo que se sugiere considerar en el análisis del programa de fortificación nacional con ácido fólico.

Entre las limitaciones de este estudio pudiese existir el sesgo de no inclusión de toda la información disponible, debido a la dificultad para el acceso a algunos artículos con texto completo, por lo que se sugiere al lector interpretar los resultados con cautela.

Para finalizar, se debe considerar en el debate, la discusión sobre beneficios y riesgos de las estrategias y programas de fortificación de ácido fólico implementada actualmente a nivel poblacional. A nivel nacional sería de utilidad realizar estudios de seguimiento a la población expuesta, ya que la bibliografía disponible corresponde a sujetos de otros países, etnia diferente y características distintas a población latinoamericana y chilena. Además es importante fomentar el monitoreo del programa de fortificación de ácido fólico con el fin de determinar dosis de fortificación seguras para todos los grupos etarios de la población.

\section{CONCLUSIONES}

De acuerdo a la bibliografía disponible, se sugiere que aún existen resultados contradictorios en relación a una elevada ingesta de ácido fólico y el aumento en el riesgo de cáncer colorrectal, y que éste riesgo está afectado por distintas variables, por lo que los autores recomiendan realizar estudios de seguimiento a nivel nacional, con el fin de establecer con mayor certeza los riesgos asociados al programa nacional de fortificación con ácido fólico.

\section{C \\ CONFLICTO DE INTERESES}

Los autores expresan que no hay conflictos de interés al redactar el manuscrito.

\section{REFERENCIAS}

(1) Ministerio de Salud, Gobierno de Chile. Norma Técnica para la Fortificación de la Harina de Trigo con Vitaminas y Minerales. 1999 p. 1-23.

(2) Ministerio de Salud, Gobierno de Chile. Decreto Supremo $N^{\circ}$ 977/96 - Reglamento Sanitario de los Alimentos. 1996 p. 1-193. 
(3) Espinosa JF. Sisvan de alimentos índices. Santiago, Chile: INTA; 1996.

(4) Hertrampf E, Cortés F. National food-fortification program with folic acid in Chile. Food Nutr Bull. 2008; 29(2 Suppl): S231-7.

(5) Llanos A, Hertrampf E, Cortes F, Pardo A, Grosse SD, Uauy R. Cost-effectiveness of a folic acid fortification program in Chile. Health Policy. 2007; 83(2-3): 295-303.

(6) Castillo C, Tur JA, Uauy R. Fortificación de la harina de trigo con ácido fólico en Chile: Consecuencias no intencionadas. Rev Med Chile. 2010; 138(7): 832-40.

(7) Hirsch S, Ronco AM, Guerrero-Bosagna C, de la Maza MP, Leiva $\mathrm{L}$, Barrera $\mathrm{G}$, et al. Methylation status in healthy subjects with normal and high serum folate concentration. Nutrition. 2008; 24(11-12): 1103-9.

(8) Terry P, Jain M, Miller AB, Howe GR, Rohan TE. Dietary intake of folic acid and colorectal cancer risk in a cohort of women. Int ] Cancer. 2002; 97(6): 864-7.

(9) Ibrahim EM, Zekri JM. Folic acid supplementation for the prevention of recurrence of colorectal adenomas: metaanalysis of interventional trials. Med Oncol. 2010; 27(3): 915-8.

(10) Castillo-Lancellotti C, Tur Marí JA, Uauy Dagach R. Suplementación con ácido fólico y prevención de recurrencia de adenomas colorrectales; revisión sistemática. Nutr Hosp. 2012; 27(1): 13-21.

(11) Qin X, Cui Y, Shen L, Sun N, Zhang Y, Li J, et al. Folic acid supplementation and cancer risk: a meta-analysis of randomized controlled trials. Int ] Cancer. 2013; 133(5): 103341.

(12) Figueiredo JC, Mott LA, Giovannucci E, Wu K, Cole B, Grainge $\mathrm{MJ}$, et al. Folic acid and prevention of colorectal adenomas: a combined analysis of randomized clinical trials. Int ] Cancer. 2011; 129(1): 192-203.

(13) Little J. Multivitamins, folate, and colon cancer. Gut. 2001; 48(1): 12-3.

(14) Sanjoaquin MA, Allen N, Couto E, Roddam AW, Key TJ. Folate intake and colorectal cancer risk: a meta-analytical approach. Int ] Cancer. 2005; 113(5): 825-8.

(15) Glynn SA, Albanes D, Pietinen P, Brown CC, Rautalahti M, Tangrea JA, et al. Colorectal cancer and folate status: a nested case-control study among male smokers. Cancer Epidemiol Biomarkers Prev. 1996; 5(7): 487-94.

(16) Mason JB, Dickstein A, Jacques PF, Haggarty P, Selhub J, Dallal $G$, et al. A temporal association between folic acid fortification and an increase in colorectal cancer rates may be illuminating important biological principles: a hypothesis. Cancer Epidemiol Biomarkers Prev. 2007; 16(7): 1325-9.

(17) Jaszewski R, Misra S, Tobi M, Ullah N, Naumoff JA, Kucuk O, et al. Folic acid supplementation inhibits recurrence of colorectal adenomas: a randomized chemoprevention trial. World ] Gastroenterol. 2008; 14(28): 4492-8.

(18) Fife J, Raniga S, Hider PN, Frizelle FA. Folic acid supplementation and colorectal cancer risk: a meta-analysis. Colorectal Dis. 2011; 13(2): 132-7.

(19) Heine-Bröring RC, Winkels RM, Renkema JMS, Kragt L, van
Orten-Luiten A-CB, Tigchelaar EF, et al. Dietary supplement use and colorectal cancer risk: a systematic review and metaanalyses of prospective cohort studies. Int ] Cancer. 2015; 136(10): 2388-401.

(20) Qin T, Du M, Du H, Shu Y, Wang M, Zhu L. Folic acid supplements and colorectal cancer risk: meta-analysis of randomized controlled trials. Sci Rep. 2015; 5: 12044.

(21) Vollset SE, Clarke R, Lewington S, Ebbing M, Halsey J, Lonn $E$, et al. Effects of folic acid supplementation on overall and site-specific cancer incidence during the randomised trials: meta-analyses of data on 50,000 individuals. Lancet. 2013; 381(9871): 1029-36.

(22) Kennedy DA, Stern SJ, Matok I, Moretti ME, Sarkar M, AdamsWebber T, et al. Folate Intake, MTHFR Polymorphisms, and the Risk of Colorectal Cancer: A Systematic Review and MetaAnalysis. J Cancer Epidemiol. 2012; 2012: 952508.

(23) Yang Z, Zhang X-F, Liu H-X, Hao Y-S, Zhao C-L. MTHFR C677T polymorphism and colorectal cancer risk in Asians, a metaanalysis of 21 studies. Asian Pac J Cancer Prev. 2012; 13(4): 1203-8.

(24) Pabalan N, Jarjanazi H, Ozcelik H. A meta-analysis of the C1420T polymorphism in cytosolic serine hydroxymethyltransferase (SHMT1) among Caucasian colorectal cancer populations. Int J Colorectal Dis. 2013; 28(7): 925-32.

(25) Lin HL, An QZ, Wang QZ, Liu CX. Folate intake and pancreatic cancer risk: an overall and dose-response meta-analysis. Public Health. 2013;127(7):607-13.

(26) Wang R, Zheng Y, Huang J-Y, Zhang A-Q, Zhou Y-H, Wang J-N. Folate intake, serum folate levels, and prostate cancer risk: a meta-analysis of prospective studies. BMC Public Health. 2014; 14: 1326.

(27) Tio M, Andrici J, Cox MR, Eslick GD. Folate intake and the risk of prostate cancer: a systematic review and meta-analysis. Prostate Cancer Prostatic Dis. 2014; 17(3): 213-9.

(28) Galeone C, Edefonti V, Parpinel M, Leoncini E, Matsuo K, Talamini $R$, et al. Folate intake and the risk of oral cavity and pharyngeal cancer: a pooled analysis within the International Head and Neck Cancer Epidemiology Consortium. Int ] Cancer. 2015; 136(4): 904-14.

(29) Zhang Y-F, Shi W-W, Gao H-F, Zhou L, Hou A-J, Zhou Y-H. Folate intake and the risk of breast cancer: a dose-response meta-analysis of prospective studies. PLoS ONE. 2014; 9(6): e100044.

(30) Li B, Lu Y, Wang L, Zhang C-X. Folate intake and breast cancer prognosis: a meta-analysis of prospective observational studies. Eur ] Cancer Prev. 2015; 24(2): 113-21.

(31) Tio M, Andrici J, Eslick GD. Folate intake and the risk of breast cancer: a systematic review and meta-analysis. Breast Cancer Res Treat. 2014; 145(2): 513-24.

(32) Liu M, Cui L-H, Ma A-G, Li N, Piao J-M. Lack of effects of dietary folate intake on risk of breast cancer: an updated metaanalysis of prospective studies. Asian Pac ] Cancer Prev. 2014; 15(5): 2323-8. 
(33) Chen P, Li C, Li X, Li J, Chu R, Wang H. Higher dietary folate intake reduces the breast cancer risk: a systematic review and meta-analysis. Br ] Cancer. 2014; 110(9): 2327-38.

(34) Zhang Y-F, Zhou L, Zhang H-W, Hou A-J, Gao H-F, Zhou Y-H. Association between folate intake and the risk of lung cancer: a dose-response meta-analysis of prospective studies. PLoS ONE. 2014; 9(4): e93465.

(35) Dai W-M, Yang B, Chu X-Y, Wang $Y-Q$, Zhao $M$, Chen $L$, et al.
Association between folate intake, serum folate levels and the risk of lung cancer: a systematic review and meta-analysis. Chin Med J. 2013; 126(10): 1957-64.

(36) $\mathrm{He} \mathrm{H}$, Shui B. Folate intake and risk of bladder cancer: a metaanalysis of epidemiological studies. Int J Food Sci Nutr. 2014; 65(3): 286-92.

(37) Tio M, Andrici J, Cox MR, Eslick GD. Folate intake and the risk of upper gastrointestinal cancers: a systematic review and metaanalysis. J Gastroenterol Hepatol. 2014; 29(2): 250-8, 